Cardiol Young 1999; 9: 663

(C) Greenwich Medical Media Ltd. ISSN 1047-9511

\title{
Diary of Events
}

\section{7-10 November 1999}

$72^{\text {nd }}$ Scientific Session of the American Heart Association, Atlanta, Georgia, USA.

Enquiries: AHA, Scientific Meetings, 7272 Greenville Ave, Dallas, TX 75231-4596. Fax: (+214) 373 3406; e-mail: scientificconferences@heart.org;

(www.americanheart.org/Scientific/confer/index.html)

12-14 November 1999

$16^{\text {th }}$ Brazilian Paediatric Cardiology Congress, Belo Horizonte-MG, Brazil.

Enquiries: Congress-Planejamento, Organização e Promoção de Eventos Ltda, 236 Francisco Sales Ave, Belo Horizonte 30150-220, Minas Gerais, Brazil. Fax: (+55 31) 2734770

\section{8-19 November 1999}

$10^{\text {ch }}$ Annual Meeting British Paediatric Cardiac Association, Belfast, N. Ireland.

Enquiries: Dr Brian Craig, Royal Belfast Hospital for Sick Children, Falls Road, Belfast BT12 6BE. Tel: $(+44)(0) 1232$ 263031

\section{8-11 December 1999}

$3^{\text {rd }}$ International Symposium on Pediatric Cardiac Intensive Care, Miami, USA.

Enquiries: David Price \& Associates, Inc, 7487 SW $50^{\text {th }}$ Terrace, Miami, Florida 33155. Fax: (+305) 663 7244; emaildpainc@compuserve.com

\section{5-7 April 2000}

$10^{\text {th }}$ Charleston Symposium on Congenital Heart Disease, Charleston, South Carolina,USA.

Enquiries: J Philip Saul, MD, Medical University of South Carolina, The Children's Heart Center, 165 Ashley Ave, PO Box 250915, Charleston, SC 29425, USA. Fax: $(+843) 792$ 3284

\section{4-17 June 2000}

$35^{\text {th }}$ Annual Meeting Association for European Paediatric Cardiology, Strasbourg, France, 14-17 June 2000. Enquiries: Dr Bernard de Geeter, Cardiologie Pédiatrique et Ftale, 3 rue Simonis, F-67100 Strasbourg. Fax: +33 (388) 3430 04; email: drdegeeter@mail.sdv.fr

\section{7-31 May 2001}

$3^{\text {rd }}$ World Congress of Paediatric Cardiology \& Cardiovascular Surgery, Toronto, Canada, May 27-31 2001. Enquiries: Dr R M Freedom, Head, Div of Cardiology, Hospital for Sick Children, University of Toronto, Faculty of Medicine, 555 University Ave, Toronto, Ont. M5G 1X8, Canada. Fax: + 1-4168137547

\section{0-11 February 2000}

Foundation Course in Cardiac Morphology, London.

Enquiries: Courses \& Conferences Office, Institute of Child Health, 30 Guilford St, London WC1N $1 \mathrm{EH}, \mathrm{UK}$. Tel: +44 (0)20 7829 8692; Fax: +44 (0)20 7831 6902; e-mail: Courses@ich.ucl.ac.uk

\section{7-20 April 2000}

$9^{\text {th }}$ Annual Symposium "Cardiology in the Young", London. Enquiries: Courses \& Conferences Office, Institute of Child Health, 30 Guilford St, London WC1N 1EH, UK. Tel: +44 (0)20 7829 8692; Fax: +44 (0)20 7831 6902; e-mail: Courses@ich.ucl.ac.uk

\section{8-20 May 2000}

International Symposium on The Developing Heart, Prague. Enquiries: Czech Medical Association J.E. Purkyne, PO Box 88, Sokolská 31, 12026 Prague 2, Czech Republic. Tel: 420 2297 271; Fax: 4202294610 ;

e-mail: senderova@cls.cz; www.biomed.cas.cz/fgu/cardiol/ dh2000.htm

\section{9-30 June 2000}

New Horizons in Fetal Cardiology, London.

Enquiries: Courses \& Conferences Office, Institute of Child Health, 30 Guilford St, London WC1N 1EH, UK. Tel: +44 (0)20 7829 8692; Fax: +44 (0)20 7831 6902; e-mail: Courses@ich.ucl.ac.uk

\section{3-14 July 2000}

Advanced Course in Cardiac Morphology, London.

Enquiries: Courses \& Conferences Office, Institute of Child Health, 30 Guilford St, London WC1N 1EH, UK. Tel: +44 (0)20 7829 8692; Fax: +44 (0)207831 6902; e-mail: Courses@ich.ucl.ac.uk 